\title{
Ajudas Externas à Memória na Intervenção em Pessoas Idosas com Comprometimento Mnésico
}

\author{
External Memory Aids in Intervention for Older People with Mnesic Impairment
}

\author{
Cristina Maria Oliveira Barbosa ${ }^{*, a}$ \& José Ignacio Guinaldo Martin ${ }^{a, b}$ \\ ${ }^{a}$ UNIFAI (ICBAS - Universidade do Porto), Porto, Portugal \\ $\&{ }^{b}$ Secção Autónoma de Ciências da Saúde, Universidade de Aveiro, Aveiro, Portugal
}

\begin{abstract}
Resumo
As estratégias de compensação são intervenções neuropsicológicas que reorganizam as funções psicológicas, incluindo as ajudas internas e externas e adaptações ambientais. As ajudas externas à memória atribuem importância a objectos externos ou ao ambiente, em prol de intervenções cognitivas ou comportamentais. O objectivo deste trabalho é analisar a eficácia e as condicionantes bio-psico-sociais inerentes à utilização das ajudas externas, por pessoas idosas com comprometimento mnésico. Procedeu-se à recolha bibliográfica de artigos científicos, maioritariamente em inglês, com critério de inclusão a intervenção em pessoas idosas, com comprometimento mnésico, e exclusão, jovens ou crianças e avaliação de outras estratégias de compensação. Os resultados sugerem que são os mecanismos compensatórios mais usados com a idade, diminuindo na demência. Devem inserir-se em sistemas de treino, ajustados às incapacidades mnésicas, potenciando a estimulação e manutenção da memória e independência.

Palavras-chave: Ajudas externas, comprometimento mnésico, pessoas idosas.
\end{abstract}

\begin{abstract}
Compensation strategies are one of the most effective ways in memory rehabilitation, including internal and external aids as well as environmental adaptations. External aids attach importance to external objects or the environment, in favor of cognitive or behavioral interventions. The purpose of this paper is to analyze the efficacy and bio-psycho-social indicators associated with the use of external aids, by older people with memory impairment. It was reviewed a variety of scientific studies, mostly in English, with inclusion criteria of intervention for older people with memory impairment. Exclusion criteria included youths or children and assessment of other compensation strategies. The findings presented them as the most commonly used compensatory mechanisms as age goes by, decreasing in dementia. It is concluded that older people should be integrated into training systems, adjusted to mnesic impairment, enhancing stimulation and maintenance of memory and independence.

Keywords: external aid, mnesic impairment, older people.
\end{abstract}

Os problemas de memória estão associados ao processo de envelhecimento normal, referido por alguns autores como Age-Associated Memory Impairment (AAMI; Crook et al., 1986 citado por Gauthier et al., 2006), a problemas patológicos, principalmente processos demenciais dos quais se destacam a Demência de Alzheimer (Lyketsos et al., 2000) ou podendo ocorrer num estádio intermédio, definido por alguns autores como Mild Cognitive Impairment (MCI; Petersen et al., 2001, citado por Burns \& Zaudig, 2002). Todas estas condições caracterizam-se por problemas associados à memória nas pessoas idosas, encontrando-se em investigação a prevalência e incidência de cada uma destas condições.

\footnotetext{
"Endereço para correspondência: Instituto de Ciências Biomédicas Abel Salazar, Universidade do Porto UNIFAI, Rua Jorge Viterbo Ferreira, 228, Porto, Portugal, 4050-313.

E-mail: cristinamobarbosa@gmail.com e jmartin@ua.pt
}

No caso da demência esta parece ser a condição com uma prevalência melhor estabelecida, mas dependente da idade das pessoas idosas, situando-se em 1,4\% para idades entre 65 a 69 anos, 2,8\% entre os 70 e 74 anos, 5,6\% dos 75 aos 79 anos, $11,1 \%$ dos 80 aos 84 anos, e de mais de $23,6 \%$ para os maiores de 85 anos (Jorm, Korten, \& Henderson, 1987). Para os casos de MCI o cálculo de prevalência parece apontar entre 3\% a 19\% da população em estudos epidemiológicos longitudinais, em países desenvolvidos (Gauthier et al., 2006). Estes dados demonstram a importância que os problemas adjacentes à memória na velhice podem ser importantes, no entanto, é em situações associadas a patologia, principalmente demência, que as dificuldades de memória são evidentemente mais fortes.

A demência caracteriza-se por perdas na capacidade de memória e por outros sintomas como afasia, agnosia, apraxia e, perturbação na capacidade de execução (American Psychiatric Association, 2000). As consequências 
da demência podem-se reflectir em incapacidades funcionais, problemas comportamentais e desordens afectivas como depressão (Koltai, Welsh-Bohmer, \& Schmechel, 2001), incapacidades ao nível da orientação espácio-temporal comportando por isso redução da capacidade de mobilidade, inibindo-se a independência e autonomia do indivíduo (Nolan, Mathews, \& Harrison, 2001).

As intervenções terapêuticas nas capacidades cognitivas de pessoas idosas com demência devem ser consideradas estratégias de reabilitação (Camp \& Skrajner, 2004), que implicam a restauração e a adaptação ao nível físico, psicológico e social, reduzindo-se o impacto das incapacidades e promovendo-se a integração social dos indivíduos (Wilson, 1997). Deve, neste sentido, obedecer a períodos de observação e de avaliação, de forma a que a intervenção reabilitadora seja eficaz e promotora de altos níveis de adaptação (De Vreese, Neri, Fioravanti, Belloi, \& Zanetti, 2001). As técnicas específicas de reabilitação da memória nas demências, baseiam-se no princípio de que a perda de memória é gradual e a sua intensidade é muito variável ao longo dos estádios (Francés, Barandiaran, Marcellan, \& Moreno, 2003). Para além disto, dada a amplitude de incapacidades causadas pelo processo demencial, a intervenção deve ser individualizada e de acordo com as necessidades, podendo ocorrer ao nível das funções cognitivas, alterações comportamentais, nível de dependência e atenção familiar (Francés et al., 2003).

A reabilitação cognitiva inclui dois tipos de estratégias: uma referente à restauração ou retraining e a outra, relativa às estratégias de compensação. Relativamente à primeira, baseia-se na teoria de que a estimulação da memória induz a recuperação, em analogia ao que acontece com a relação entre exercício físico e melhoramento contínuo da capacidade física (Turkstra, 2001). Este trabalho focase na segunda - compensação, que normalmente, corresponde a estratégias ou processos através dos quais os indivíduos podem-se adaptar às incapacidades de memória (Bäckman \& Dixon, 1992; De Frias, Dixon, \& Bäckman, 2003), ou seja, são acções que tratam de alterar as perdas ou incapacidades das características ou estados dos indivíduos; incluindo-se a (a) remediação, implicando um maior investimento de tempo e esforço; (b) substituição, com o desenvolvimento de novas ou latentes competências; (c) acomodação, através do ajustamento de objectivos e critérios consoante as competências e (d) assimilação, pela modificação ambiental e/ou expectativas (Dixon, De Frias, \& Bäckman, 2001). Os comportamentos compensatórios usados pela população idosa incluem várias estratégias, sendo estas a (a) manutenção do sucesso e competência profissional ou do dia-a-dia; (b) acomodação às perdas pessoais ou sociais; (c) activação ou recrutamento de novas regiões neuro-anatomicas relacionadas com a performance nas tarefas; (d) superação dos défices cognitivos ou sensoriais; (e) reabilitação ou ajustamento comportamental às incapacidades ou lesões neurológicas (De Frias et al., 2003).
As estratégias de compensação são actualmente a intervenção mais eficaz na recuperação mnésica, incluindo nesta as (a) ajudas internas (tais como técnicas de associação visuo-espacial na recordação); (b) as adaptações ambientais (como modificações arquitectónicas e ambientais) e por último, (c) as ajudas externas - diários, bloco de notas, listas e organizadores electrónicos (Evans, Wilson, Needham, \& Brentnall, 2003).

As ajudas externas à memória, como o uso de caixas de medicação para lembrar a toma de medicamentos ou um alarme para lembrar de consultar a lista diária, são uma das melhores estratégias de compensação, sendo utilizadas durante bastante tempo, por um grande número de indivíduos (Wilson \& Watson, 1996). Esta opinião é ainda corroborada por Hodges et al. (2006), referindo que as ajudas externas apoiam os indivíduos na compensação dos défices de memória, pensando-se que seja uma das ajudas de reabilitação mais eficazes. Na opinião de Wilson (1997), o uso de ajudas externas de forma eficaz envolve a memória, e neste caso, muitos indivíduos têm dificuldade em recorrer a estas. Esta opinião é apoiada por Dixon, Hopp, Cohen, De Frias e Bäckman (2003), que referem que os indivíduos saudáveis usam mais frequentemente as ajudas externas do que os indivíduos com demência. Em contrapartida a opinião de Nolan et al. (2001), aponta para que os indivíduos com demência, em estados moderados e aqueles com lesão cerebral, como sendo os que preferencialmente usam ajudas externas.

Este trabalho procura analisar estas variantes e percepcionar quais as condicionantes bio-psico-sociais adjacentes ao uso de ajudas externas à memória pela população idosa com incapacidades mnésicas, e desta forma elaborar uma análise crítica de trabalhos científicos, relativos à utilização de ajudas externas em indivíduos adultos ou idosos com problemas mnésicos.

Evans et al. (2003) investigaram os factores preditores ao uso de estratégias de compensação, chegando à conclusão de que os principais são: idade, em que os jovens compensam de melhor forma; severidade do comprometimento - indivíduos com graves incapacidades usam menos as estratégias de compensação; especificidade do défice - indivíduos com défices cognitivos generalizados parecem compensar menos do que aqueles com défices mais focalizados e uso prévio de estratégias compensatórias - aqueles que usavam estratégias, parecem usar melhor após uma lesão cerebral ou incapacidade.

As perdas cognitivas, especificamente aquelas relativas à memória, causam problemas de manutenção de independência no dia-a-dia. Segundo Lowenstein et al. (1992, 1995, citado por McDougall, 1998) o sexo feminino é mais vulnerável a problemas associados com a independência em actividades da vida diária, quando associados a maior idade, depressão e perda progressiva de memória. Na sua maioria, as pessoas idosas querem manter as suas competências mnésicas através do aumento da memória do dia-a-dia: nomes de pessoas, datas importantes, números de telefone, objectos importantes, eventos re- 
centes e passados, anotações, informações e factos e direcções (Bolla et al., 1991; Leirer et al., 1990, citados por McDougall, 1998). Neste sentido, as estratégias de compensação, em particular as ajudas externas, são as mais ajustadas ajudando os indivíduos a relembrar as suas anotações, a tomar a medicação e realizar outras tarefas relacionadas com a independência no dia-a-dia (Hodges et al., 2006). As ajudas externas têm demonstrado serem as melhores estratégias compensatórias em situações de problemas de memória, organizacionais e de planeamento (Wilson, Emslie, Quirk, \& Evans, 2001), traduzindo-se assim num tipo de técnica que aposta na promoção das competências do dia-a-dia e na qual está, normalmente, implícita os vários tipos de memória.

De uma forma geral no dia-a-dia, os indivíduos sem comprometimento cognitivo recorrem ao uso de ajudas externas, como são as anotações pessoais diárias (Tran, Mynatt, \& Calcaterra, 2007). Durante o processo normal de envelhecimento, bem como em casos de lesões cerebrais ou estádios iniciais de patologias neurodegenerativas, os indivíduos continuam com a capacidade de aprendizagem, assim como espontaneamente de implementarem técnicas de compensação dos declínios e défices da memória (Dixon \& De Frias, 2007).

Especificamente na intervenção junto de pessoas idosas com demência, têm-se vindo a desenvolver trabalhos, que pretendem percepcionar a eficiência de metodologias baseadas na compensação por ajudas externas. Segundo Bourgeois et al. (2003), o estudo da conversação de indivíduos que recorrem ao uso de livros de memória como ajuda externa, revelou nestes, frases mais elaboradas e menor ambiguidade e repetições, quando comparada com situações onde não se recorria a ajudas externas, como estratégia de compensação mnésica.

A reabilitação cognitiva pode recorrer a dispositivos prostéticos, que funcionam como substitutos da função da memória, nomeadamente relógios alarme, relógios, sinais, calendários e cartões, dispositivos ortóticos, que optimizam a função da memória, como o caso de softwares de computadores ou telefones automáticos e ambientais, quando fazem parte do ambiente, como os sinais ou portáteis, podendo ser mudados de localização, como são os cadernos de apontamento ou organizers (Bourgeois, 2006).

As ajudas externas são referidas como "próteses cognitivas" que envolvem o uso de ajudas tangíveis e físicas externas aos sujeitos (Soler \& Ruiz, 1996). Bourgeois (2006) afirma que as ajudas externas, em oposição às internas, podem existir no meio físico sendo consideradas como próteses de memória, podendo-se mencionar vários exemplos tais como livros de notas, notas com lembranças, escrita na mão, notas no calendário, mudanças físicas para criar pistas, temporizadores, colocar algo sempre no mesmo sitio ou num sitio especifico, pedir a alguém que nos lembre de algo, atribuir significados (Soler \& Ruiz, 1996).
Em alguns casos, as ajudas externas podem-se associar a equipamentos de uso diário no apoio à vida diária da pessoa idosa (Turkstra, 2001), como pode ser o caso dos temporizadores (ajuda externa) no controlo do fogão (equipamento).

Na opinião de Wilson \& Watson (1996), há uma acentuada aceitação desta estratégia compensatória, pois é facilitadora da recuperação da informação armazenada internamente (através de lembretes de data especiais, é capaz de se lembrar). Por outro lado, as ajudas externas possibilitam a recuperação de informação pela própria memória, ou seja, o indivíduo de forma autónoma e independente através de por exemplo, relógios de maior dimensão, calendários, quadros de informação, recorda-se dos factos (Woods, 1994).

Os indivíduos com incapacidades mnésicas, costumam apresentar alguns problemas no uso regular de ajudas externas devido, (a) ao seu esquecimento; (b) à incapacidade de programação das ajudas (no caso de recorreram a tecnologia); (c) ao uso sistemático e rotineiro da ajuda externa que provoca habituação; e por último (d) ao embaraço (social) no seu uso (Wilson, Evans, Emslie, \& Malinek, 1997). Para além disto, apontam-se ainda razões relacionadas com o demasiado tempo de utilização, associado ao cansaço de uso, os constrangimentos financeiros, alterações psicossociais e a frustração, como factores potenciadores de dificuldades na utilização das ajudas externas, pelos indivíduos e pelas suas famílias (Wilson et al., 1997).

As ajudas externas à memória representam um avanço nas metodologias de intervenção terapêutica e de relação doente-clínico, sendo estratégias de intervenção não farmacológica, de utilização transversal a várias idades, contextos e comprometimentos. Traduz-se ainda do ponto de vista social numa oportunidade de responsabilização individual perante o processo terapêutico e de consciencialização social da importância da retaguarda (in)formal de apoio do doente. Assim poderá afirmar-se ser uma ferramenta parcimónia, ecológica e promotora de estimulação contínua.

\section{Método}

Várias metodologias foram usadas no sentido de pesquisar e reunir trabalhos de investigação, cuja temática, se relacionasse com o objectivo do presente artigo. A primeira metodologia prendeu-se com a pesquisa avançada e recolha de informações no período de Dezembro de 2008 e Abril de 2010, na Medline usando-se como palavras-chave, ajudas externas (external aids), pessoas idosas (older people), Demência (Dementia), alterações de memória (memory impairments). As várias publicações pesquisadas, desde artigos científicos e livros, foram maioritariamente no idioma inglês, no entanto também se pesquisou em português e espanhol, tendo os trabalhos como período de publicação entre 1987 e 2009. 
De forma a focalizar o presente trabalho, sobre o impacto que as ajudas externas têm em pessoas idosas com demência procedeu-se à selecção dos vários trabalhos realizados nesta área, usando-se critérios de inclusão e de exclusão dos mesmos. Os critérios de inclusão foram a amostra constituir-se total ou parcialmente com indivíduos adultos ou idosos, com diagnóstico demencial ou outro tipo de comprometimento mnésico e em que sejam avaliadas estratégias de compensação, em particular as ajudas externas. Os critérios de exclusão, foram aplicados a artigos com intervenções em jovens ou crianças e avaliação de outras estratégias de compensação, que não incluísse ajudas externas.

Tabela 1

Estudos Exploratórios Relativos à Utilização de Ajudas Externas nas Pessoas com Problemas Mnésicos

\begin{tabular}{|c|c|c|c|}
\hline Fonte & Objectivos & Amostra & Resultados \\
\hline $\begin{array}{l}\text { Reese Cherry, } \\
\text { \& Norris } \\
(1999)\end{array}$ & $\begin{array}{l}\text { (a)Descrever as } \\
\text { preocupações dos } \\
\text { idosos com a memória }\end{array}$ & $\begin{array}{l}N_{(1)}=29 \%_{(2)} \stackrel{+}{(3)}=79,3 \\
100 \% \text { inf ao Ens. Sec. }\end{array}$ & $\begin{array}{l}\text { (a) Ajudas externas são a estratégia de memória mais utili- } \\
\text { zada. }\end{array}$ \\
\hline
\end{tabular}
(2001)
Dixon et al. (a) Avaliar, pelo $\mathrm{MCQ}_{(4)}$ as estratégias de compensação da memória no dia-a-dia Identificar propriedades psicométricas do MCQ
$N_{\text {total }}=854 \mid n_{1}=331$ $n_{2}=523 X_{(5)}$ Idade 1 $=71,\left.45(5,53)\right|_{\text {Idade } 2} ^{\text {Idade }}=$ $68,26(7,31) n$ 우 $_{1}=200$ | 우 $_{2}=350$

(a) As ajudas externas são o mecanismo compensatório mais usado entre os idosos do estudo $(X=3,3)$. (b) Na escala de ajudas externas existe um efeito entre a idade e género, sendo que os homens idosos referem um melhor uso de ajudas externas do que os homens jovens, enquanto as mulheres referem uma frequência similar no uso de ajudas externas $\left(X{ }^{\circ}\right.$ idosas-jovens $=3,4 \mid{ }^{\circ}$ idosas-idosas $\left.=3,4\right)(\mathrm{c})$ As mulheres mostram valores superiores de motivação, do que os homens $\left(X\right.$ 우 $\left.=2,01 ; X 0^{x}=1,76\right)$
Dixon et al. (2003) (a) Avaliar pelo MCQ as técnicas de compensação de memória $2_{\text {grupos }}\left|\mathrm{n}_{\mathrm{ctl}}=85\right| \mathrm{n}_{\mathrm{DA}(6)}=$ $2 \stackrel{1}{1} X_{\text {idade }} \mathrm{ctl}=81,8(3,1)$ $\left.\right|_{\mathrm{DA}}=81,8(2,8) \%$ ㅇ $_{\mathrm{ctl}}=$ $77,\left.6\right|_{\mathrm{DA}}=61,9 X$ $\stackrel{\mathrm{ctl}}{=} 9,9(3,8) \stackrel{\mathrm{DA}}{\mathrm{D}_{\mathrm{DA}}}=$ $8,8(3,9) X_{\mathrm{MMSE}(7) \mathrm{ctl}}=$ $28,\left.3(1,9)\right|_{\mathrm{DA}} ^{\mathrm{MMSE}(7)} 23,6$ $(3,5)$ (a) Os indivíduos saudáveis e com DA apresentam uma frequência similar de comportamentos de compensação. (b) $\mathrm{O}$ uso de ajudas externas é o mais referido mecanismo de compensação, com diferença estatisticamente significativa entre os indivíduos saudáveis (maior utilização) do que os indivíduos com DA (menor utilização). (c) As ajudas externas são uma das estratégias mais fáceis de serem usadas por indivíduos com alterações de memória.
Evans et al. (a) Prever o uso (2003) eficaz de ajudas de memória (internas e externas)
$N=94$ (pessoas idosas pós-lesão cerebral) $X_{\text {idade }}=39,5(13,4) \mid \%$ ㅇ $=31,9 \mid X_{\text {anos educação }}=$ $12,0(2,1)$ (a) De entre as ajudas de memória, as ajudas externas são as mais usadas, sendo que a mais usada é o calendário de parede/quadro de parede $(n=68)$ e a menos é a combinação relógio/calendário $(n=1)$. O uso de ajudas electrónicas é reduzido $(n=7)$. (b) As ajudas mais usadas não são necessariamente as mais eficazes (na opinião dos cuidadores). (c) Comprova-se a utilização de ajudas externas como mecanismo de compensação, uma vez que a utilização de ajudas externas antes da lesão, é inferior à sua utilização após.

\begin{tabular}{|c|c|c|c|}
\hline $\begin{array}{l}\text { De Frias } \\
\text { et al. } \\
(2003)\end{array}$ & $\begin{array}{l}\text { (a) Relacionar } \\
\text { indicadores de saúde } \\
\text { e psicossociais, } \\
\text { com o uso de } \\
\text { estratégias } \\
\text { compensatórias } \\
\text { de memória em } \\
\text { pessoas idosas }\end{array}$ & $\begin{array}{l}N=528 X_{\text {idade }}= \\
68,50(7,6) \mid n \text { ㅇ }=355 \mid \\
X_{\text {educação }}=14,8(3,1)\end{array}$ & $\begin{array}{l}\text { (a) A idade está associada a maior comprometimento da } \\
\text { memória e maior uso de estratégias de compensação(b) As } \\
\text { mulheres referem maior uso de estratégias externas e inter- } \\
\text { nas e um maior esforço em lembrarem-se, enquanto os ho- } \\
\text { mens confiam mais noutras pessoas, como ajudas de me- } \\
\text { mória. (c) Um maior uso de estratégias de compensação está } \\
\text { associado a um maior número de patologias, assim como } \\
\text { uma utilização mais frequente de ajudas externas está asso- } \\
\text { ciada a uma maior percepção de défices e afectos negativos. } \\
\text { (d) Uma baixa pontuação no } \text { MSE }_{(8)} \text { está associado a maior } \\
\text { uso de ajudas externas ou outras pessoas como apoio. }\end{array}$ \\
\hline
\end{tabular}




\begin{tabular}{|c|c|c|c|}
\hline $\begin{array}{l}\text { Camp \& } \\
\text { Skrajner } \\
(2004)\end{array}$ & $\begin{array}{l}\text { Determinar } \\
\text { os efeitos da } \\
\text { implementação } \\
\text { do Programa } \\
\text { Montessori em } \\
\text { residências } \\
\text { assistidas } \\
(\text { RAMP) } \\
(9)\end{array}$ & $\begin{array}{l}N_{\mathrm{T}}=13\left|\mathrm{n}_{\text {lideres }}=4\right| n_{\text {jogadores }}= \\
9 X_{\text {idade }}=84 \mid \\
X_{\text {idade lideres }}=80 \mid X_{\text {idade jogado- }} \\
\stackrel{=}{=}=88,7(4,1) \mid X_{\text {MMSE líderes }} \\
23 \mid X_{\text {MMSE jogadores }}=8,3\end{array}$ & $\begin{array}{l}\text { (a) Através do uso de ajudas externas foi desenvolvido um } \\
\text { protocolo de procedimentos de actuação, para apoiar os } \\
\text { líderes;(b) Um dos procedimentos que necessitou de apoio } \\
\text { do staff foi para lembrar os jogadores de colocarem as car- } \\
\text { tas vencedoras na caixa;(c) Um dos procedimentos mais } \\
\text { desafiantes para os líderes foi p.ex: instruir os jogadores } \\
\text { para iniciar uma discussão aberta;(d) Os jogadores respon- } \\
\text { dem bem à RAMP, sugerindo um envolvimento positivo e } \\
\text { afectivo durante o RAMP }\end{array}$ \\
\hline $\begin{array}{l}\text { Dixon } \\
\text { \& De Frias } \\
(2007)\end{array}$ & $\begin{array}{l}\text { (a) Analisar estraté- } \\
\text { gias de compensa- } \\
\text { ção de memória } \\
\text { usadas por idosos } \\
\text { de dois grupos: } \\
\text { Grupo NIC }_{(10)} \text { (sem } \\
\text { défice de memoria) } \text { MMD }_{(11)} \text { (défice } \\
\text { médio de memória), } \\
\text { ao longo de } 6 \text { anos }\end{array}$ & 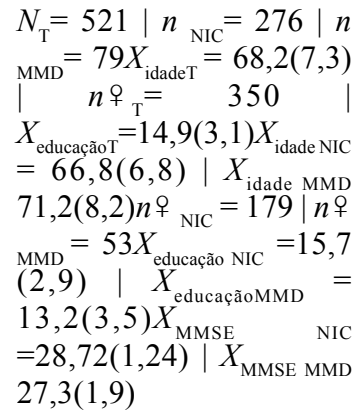 & $\begin{array}{l}\text { (a) } \mathrm{O} \text { estado da memória é um preditor significativo nas } \\
\text { diferenças inter-individuais nas ajudas externas }\left(\mathrm{g}_{10}=0,06 \text {, }\right. \\
=-0,08) \text {, assim como no esforço investido }\left(\mathrm{g}_{10}=0,05, \mathrm{~g}_{13}=\right. \\
\left.{ }_{13}=0,10\right) \text {.(b) Os grupos NIC e MMD divergem no uso e no } \\
\text { esforço para manter a ajuda externa (NIC aumenta e MMD } \\
\text { diminui).(c) O grupo MMD refere maior recorrência a ou- } \\
\text { tras pessoas como assistência de memória. }\end{array}$ \\
\hline
\end{tabular}

Notas. ${ }^{(1)} N$ ou $n$-tem um número de sujeitos; ${ }^{(2)} \%$ - percentagem; ${ }^{(3)}$ - sexo feminino; ${ }^{(4)}$ - MCQ - Questionário de Compensação da Memória; ${ }^{(5)}$ - X- média de determinado factor; ${ }^{(6)}$ - DA - Demência de Alzheimer; ${ }^{(7)}$ - MMSE - Mini Mental State Examination; (8) - MSE - questionário de auto-eficácia da memória; ${ }^{(9)}$ - RAMP - Baseia o seu treino em pessoas (líderes e jogadores) com demência inicial, através de várias actividades, com recurso intensivo a ajudas externas; ${ }^{(10)}$ - NIC - Sem comprometimento cognitivo; (11) - MMD - Médio comprometimento cognitivo.

Tabela 2

Estudos de Intervenção Relativos à Utilização de Ajudas Externas nas Pessoas com Problemas Mnésicos

Fonte Objectivos Amostra Resultados

O'Hanlon, ração espaçada e os calen-

\& Stevens dários comlembranças

(1996) escritas, aumentam nos

indivíduos com DA, a

performance da memória

prospectiva (b) Determinar

se os efeitos do treino com

calendário, permanecem 6

meses após a intervenção

(c) Avaliar os factores que

contribuem para o uso com

sucesso do calendário
Camp, Foss, (a) Determinar se a recupe- $\quad N=5$

$\begin{array}{ll}\text { McDougall } & \text { (a) Testar a eficácia do } \\ \text { (1998) } & \text { CBMEM }\end{array}$ do uso de estratégias de memória internas e externas (b) Melhorar a auto-eficácia da memória

\section{Clare et (a) Investigar se os}

al. (2000) princípios de "aprendizagem sem erros" são eficazes na intervenção dos problemas de memória, em estádios iniciais de DA

(b) Avaliar a intervenção baseada no princípio da (Re)Aprendizagem
$N_{\mathrm{T}}=33 \quad\left|N_{\text {exp }}=23\right|$

$N_{\text {comp }}^{\mathrm{T}}=10 X_{\text {idade }}=$ $69,48(5,82) \mid n$ 우 $=25 \mid$

$X_{\text {educação }}=5 \mid X_{\mathrm{MMSE}}=25$

(a) Não se verificaram diferenças significativas no uso global das ajudas externas entre o pré e pós teste(b) Houve aumento significativo no uso de listas $(2,55 \mathrm{vs}$ $3,38)$ e de notas $(3,27$ vs 3,75), entre o pré e o pós teste(c) Não houve diferenças no uso de calendário, sitio ou outros, entre o pré e o pós teste

$N=6$ (estádios iniciais de demência de Alzheimer) $X_{\text {idade }}=$ $69,3(3,9) \mid n$ 우 $=3 \mid$ $X_{\mathrm{MMSE}}=24(2,1)$

(a) Os participantes A, B, C, D aumentaram os seus valores, na e após intervenção (b) $\mathrm{O}$ participante $\mathrm{E}$, mostrou uma redução da frequência de questões repetitivas a seguir à intervenção(c) Não se verificou esta redução no participante $\mathrm{F}(\mathrm{d}) \mathrm{A}$ participante $\mathrm{E}$, manteve as suas melhorias no follow-up e continuou a usar a ajuda externa de memóriaNo participante F, o uso da ajuda externa nunca se tornou firme, tendo deixado de a usar após a intervenção [Part. A - 
aprendizagem de 11 nomes de participantes num clube; usaram fotos, repetição verbal, facial | Part. B aprendizagem de 13 nomes; usaram elaboração verbal baseada na facial, fotos, | Part. C - nomes de pessoas famosas, reconhecimento facial, sinais, | Part. D - Lista de informações pessoais e treino com recuperação espaçada, usando áudio | Part. E - uso de calendário e quadro de memoria, no planeamento diário | Part F estratégia de memória - NeuroPage]

$\begin{array}{lll}\text { Koltai et al. } & \begin{array}{l}\text { (a) Avaliar os efeitos de um } \\ \text { programa de memória e }\end{array} & N=24 X_{\text {idade }}=72,9(6,7) \\ & \begin{array}{l}\text { coping }(\mathrm{MCP})_{(2)}, \text { em idosos } \\ \text { com demência moa }=15,0(4,0)\end{array} & X_{\mathrm{MMSE}}=22,9(3,6) \\ & \end{array}$

(a) Há diferenças estatisticamente significativas $(p=0,028)$ de ganhos no funcionamento da memoria perceptiva, com e sem insight(b) $\mathrm{O}$ insight aumenta a percepção de ganhos entre os participantes(c) O insight é uma variável importante na fase de intervenção/tratamento
Nolan et al. (2001) (a) Avaliar o impacto de duas ajudas externas (foto e nome), na localização do quarto de pessoas idosas com DA
$N=3 X_{\text {idade }}=86 \mid n$ o $=$ $100 \%\left|X_{\text {MMSE A }}=7\right|_{\mathrm{B}}=$ $\left.4\right|_{\mathrm{C}}=6$

(a) A associação das duas ajudas externas aumenta 50\% da capacidade das pessoas idosas localizarem o seu quarto(b) $X_{\text {fase de intervenção }}=85 \%$ (c) $X_{\text {pós-intervenção }}=50 \%$

$\begin{array}{lll}\text { Bottino et } & \text { (a) Avaliar os resultados de } & N=6 X_{\text {idade }}=74,67(6,98) \\ \text { al. (2002) } & \text { um tratamento combinado } & \mid n+1 \\ & \text { (inibidor de acetilcolineste- } & X_{\text {educação }}=7,50(3,98) \\ & \text { rase }+ \text { treino cognitivo) em } \\ \text { pessoas idosas com DA } & X_{\text {MMSE }}=23,17(3,43) \\ & & \end{array}$

(a) As ajudas externas podem ser adaptadas a pessoas idosas com demência, demonstrando melhorias cognitiva e funcional(b) A associação de técnicas de reabilitação cognitiva à medicação, auxilia na estabilização ou leve melhoria dos défices cognitivos e funcionais(c) Importante associar medicamentos com equipas multi-disciplinares

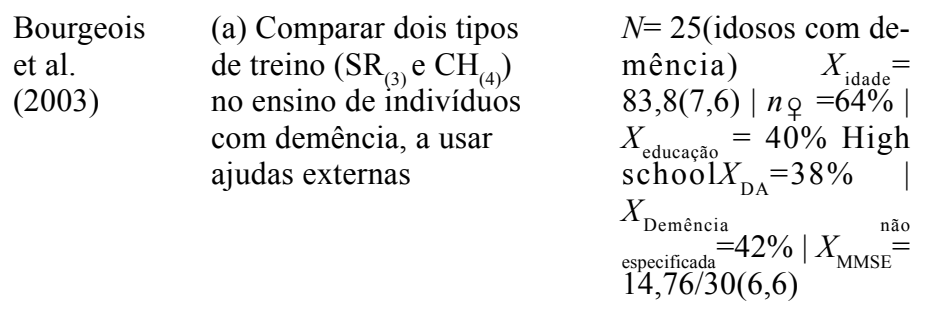

(a) Há diferenças estatisticamente significativas $(p<0,035)$ ao atingir os objectivos: $\mathrm{SR}=23$ participantes; $\mathrm{CH}=18$ participantes (b) SR apresenta mais vantagens (c) Não há diferenças estatisticamente significativas no número de sessões requeridas em ambas as intervenções (d) Indivíduos com demência podem adquirir novas aprendizagens, usando ambos os treinos (e) As ajudas externas podem ser usadas por indivíduos com demência, desde que sujeitos a treino específico

$\begin{array}{ll}\text { Melton \& } & \text { (a) Investigar a eficácia da } \\ \text { Bourgeois } & \text { SR, no ensino de indivíduos } \\ \text { (2005) } & \begin{array}{l}\text { com traumatismo craniano, } \\ \text { usando o telefone. }\end{array}\end{array}$

Tran et al. (2007)
$N=7$ (pessoas idosas com lesões de traumatismo craniano) $X_{\text {idade }}=$ $47 \mid n$ o $=3 \mid$ BTHI $_{(5)}=$ 3 severidade media; 4linha do normal

(a) A SR é viável no treino de uso de ajudas externas à memória (b) O telefone, enquanto ajuda externa, deve ser considerado como um serviço a promover na população com lesão cerebral (c) Na pós intervenção houve $77,7 \%$ de continuação do uso de ajudas de memória para a realização de tarefas

$N=3$ Jovens adultos $n$ o $=2$ (a) O uso de ajuda externa promove independência da memória e auto-confiança

(a) O uso de ajuda externa promove independência da memória e auto-confiança ria na cozinha, provoca dependência no seu uso

$$
\begin{aligned}
& N_{\text {total }}=40 \mid N_{\text {MCI (6)controlo/ }} \\
& \text { MMSE }=12 / 28,0 \mid N_{\text {MCI }} \\
& \text { intervenção/MMSE } \\
& \mid N_{\text {Mild Dementia intervenção/ }} \\
& =18 / 27,8 \\
& \text { MMSE } \\
& n_{\text {o total }}=22 \mid \\
& X_{\text {idade total }}=69 \mid X_{\text {educą̧ão }} \\
& 9,8 \mid
\end{aligned}
$$

(a) Explorar os benefícios Ramsenthaler, de um programa de \& Sorg reabilitação cognitiva, (2009) com multi-componentes, em 3 grupos de participantes (a) $\mathrm{O}$ uso de ajudas externas à memória foi uma das componentes de intervenção do treino cognitivo; (b) Através de trabalhos manuais e criativos, os participantes elaboraram as suas ajudas externas; (c) No grupo de intervenção MCI, houveram melhoramentos com diferenças estatisticamente significativas em todas as variáveis: memória episódica verbal; memória episódica não verbal; realização de actividades da vida diária; (d) No grupo de Mild Dementia não houve melhoramento significativo nestas variáveis;

Notas. (1) CBMEM - Modelo cognitivo-comportamental da memória do dia-a-dia; (2) MCP - Inclui treino nas seguintes áreas: recuperação espaçada; estratégias de reconhecimento cara-nome; elaboração verbal; concentração/repetição; ajudas externas; estratégias de coping; ${ }^{(3)} \mathrm{SR}$ - Recuperação espaçada; ${ }^{(4)} \mathrm{CH}$ - Hierarquia de pistas; ${ }^{(5)} \mathrm{BTHI}$ - Bateria de testes de avaliação de traumatismo craniano; ${ }^{(6)} \mathrm{MCI}$ - síndrome de declínio cognitivo. 
Tabela 3

Estudos de Intervenção Relativos à Utilização de Ajudas Externas com Recurso a Tecnologia, nas Pessoas com Problemas Mnésicos

\begin{tabular}{|c|c|c|c|}
\hline Fonte & Objectivos & Amostra & Resultados \\
\hline $\begin{array}{l}\text { Wilson et } \\
\text { al. (1997) }\end{array}$ & $\begin{array}{l}\text { (a) Avaliar a } \\
\text { eficácia do } \\
\text { NeuroPage }_{(1)} \text { como } \\
\text { ajuda externa, nas } \\
\text { falhas da memoria } \\
\text { do dia-a-dia }\end{array}$ & $\begin{array}{l}N=15 X_{\text {idade }}=40|n+=4| \\
10 \text { com diagnóstico de } \\
\text { lesão cerebral }\end{array}$ & $\begin{array}{l}\text { (a) } \text { Sucesso }_{\text {base-line }}=37,08 \% \mid \text { Sucesso } \\
\text { Sucesso } \\
\text { follow-upento } \\
=74,46 \% \text { - demonstra claramente um aumento } \\
\text { significativo das tarefas realizadas com sucesso (b) Melhorias } \\
\text { superiores na fase de follow-up, quando comparada com a fase } \\
\text { de base-line (c) No follow-up existe uma grande diferença entre } \\
\text { participantes - para alguns não há declínio da performance, } \\
\text { para outros há quebras acentuadas }\end{array}$ \\
\hline
\end{tabular}

\begin{tabular}{|c|c|c|}
\hline $\begin{array}{l}\text { Wilson et } \\
\text { al. }(2001)\end{array}$ & $\begin{array}{l}\text { (a) Avaliar um } \\
\text { sistema de pager } \\
\text { na capacitação nas } \\
\text { tarefas diárias }\end{array}$ & $\begin{array}{l}N_{\mathrm{T}}=143 \mid \\
38,57(14,87) \mid n+=38 \\
44,1 \% \text { com traumatismo } \\
\text { cranianoApós estudo pi- } \\
\text { loto divide-se em } 2 \text { gru- } \\
\text { pos: A e B | A - recebe o } \\
\text { pager logo após a avali- } \\
\text { ação | B - recebe pager } \\
\text { após } 7 \text { semanas }\end{array}$ \\
\hline
\end{tabular}

Hodges et (a) Testar a utiliza- Estudo de caso $(N=1)$ al. (2006) ção de SenseCam ${ }_{(3)}$, Sujeito com 63 anos; como ajuda externa, sexo feminino; bom nível na memória educacional; diagnóstico retrospectiva de alterações mnésicas (a) Não há diferenças estatisticamente significativas no cumprimento dos objectivos entre os dois grupos, em relação à idade, tempo com lesão, género, (b) $84,6 \%$ dos participantes completaram as tarefas, com melhorias significativas, utilizando o Sistema de Pager (c) 4,9\% apresenta melhoria entre fases; $6,3 \%$ não mostra diferenças; $4,2 \%$ ficaram piores com o Sistema de Pager

(a) Com o SenseCam, a utente recorda mais aspectos de vários eventos(b) Melhoria na memória auto-biografica(c) A participante recorda três vezes mais detalhes, durante o período regular de revisão(d) Com o uso de SenseCam, provocou na utente um sentimento de confiança, diminuindo sintomatologia ansiosa, em eventos sociais

Notas. ${ }^{(1)}$ NeuroPage - Sistema de pager portátil; ${ }^{(2)}$ Sistema de Pager - Ajuda electrónica que avisa/alerta para determinadas tarefas, anteriormente programadas; ${ }^{(3)}$ Sense Cam - Câmara desenvolvida com um sensor capaz de identificar momentos propícios para fotos.

\section{Resultados}

Partindo da análise dos estudos apresentados poderão inferir-se alguns dados relativos às ajudas externas e particularidades da sua utilização. Neste estudo teve-se essencialmente por base a pesquisa através de artigos científicos, sendo seleccionados 33 artigos, dos quais 20 foram incluídos na análise de resultados, e os restantes enquadraram-se em apoio teórico-pratico. De forma a diversificar as várias visões que apoiam a utilização e enquadramento das ajudas externas como metodologia de intervenção, foi realizada uma pesquisa bibliográfica bastante diversificada, rebuscando em diferentes trabalhos científicos múltiplas visões e aplicações das ajudas externas e enquadramento dos seus utilizadores. Assim, embora não com grande expressividade, poderá assinalar-se a revista Journal of Clinical and Experimental Neuropsychology como sendo a mais predominante, como fonte de três artigos utilizados. Da análise dos 20 estudos infere-se um total de 2456 participantes, com uma média de 123 participantes por estudo, idade média de 67,9 anos com $61,2 \%$ dos indivíduos do sexo feminino (1482 participantes). Para além disto, poderá apontar-se cinco tipos de trabalhos científicos encontrados, e que contribuíram para a pesquisa aprofundada deste tema. Na sua maioria os artigos relacionam o uso de ajudas externas à memó- ria: (a) com demência $(n=12)$, dos quais 7 avaliaram esta questão num contexto associado à demência de tipo Alzheimer; (b) com problemas de memória $(n=8)$; (c) com lesões cerebrais $(n=2)$; (d) perdas mnésicas associadas ao processo normal de velhice $(n=6)$ e os restantes apoiaram a pesquisa teórica $(n=5)$.

Vários indicadores parecem influenciar o uso de estratégias de compensação, especificamente as ajudas externas. Os estudos apontam para que as ajudas externas sejam mais utilizadas com o aumento da idade (De Frias et al., 2003; Dixon et al., 2001), constituindo-se como uma forma eficaz de reabilitação (Hodges et al., 2006), sendo a estratégia de compensação mais utilizada (Dixon et al., 2001; Reese et al., 1999), mas não necessariamente a mais eficaz (Evans et al., 2003). Relativamente à relação entre o estado de memória e o uso de ajudas externas, Dixon et al. (2003) referem que os indivíduos sem comprometimento aumentam o uso das ajudas externas, por outro lado, Grandmaison e Simard (2003), concluem que em estádios iniciais de demência de Alzheimer, aumenta de forma espontânea o uso de ajudas externas, ao passo que na opinião de Dixon \& De Frias (2007), com comprometimento médio, reduzem o uso de ajudas externas. Assim, verifica-se que o estado da memória poderá predizer o uso individual de ajudas externas, no entanto, este indicador deverá ser melhor estudado, pois Dixon et al. 
(2003), concluíram que os indivíduos saudáveis e com Doença de Alzheimer (DA), referem similar frequência de estratégias de compensação.

As ajudas externas, apresentam resultados eficazes, mesmo em sujeitos com demência (Bourgeois et al., 2003) e estas conclusões são corroboradas pelos estudos de Bottino et al. (2002) e de Tran et al. (2007), que apontam no sentido das ajudas externas proporcionarem independência, aumento da auto-estima e da auto-confiança dos indivíduos.

Do ponto de vista do estado de saúde, as polipatologias, associam-se também ao maior uso de estratégias compensatórias (De Frias et al., 2003), assim como o maior insight perante a sua situação e as suas incapacidades (De Frias et al., 2003). Do ponto de vista psicossocial, os afectos negativos associam-se ao maior uso de ajudas externas e em contrapartida resultados baixos no MSE (Memory Self-Efficacy - questionário de auto-eficácia da memória; avalia a auto-capacidade em memorizar) estão associados a um maior uso de ajudas externas, tendo neste contexto influência a percepção que o individuo tem da auto-eficácia da sua memória. Verifica-se que quanto mais baixa for a percepção da capacidade de relembrar, maior é a tendência do uso de ajudas externas (De Frias et al., 2003).

Relativamente ao género, o sexo feminino apresenta valores superiores de motivação e de uso de ajudas externas, quando comparadas com o sexo masculino, recorrendo estes mais ao apoio de outrem nas questões de lembrança (Dixon et al., 2001). Poderão estes factos apontar para um comportamento mais independente das mulheres, demonstrando maior investimento na sua independência.

A intervenção junto de pessoas idosas com demência deve incluir treinos específicos e contínuos (Bourgeois, 2006), aliada ao cruzamento de técnicas (Bourgeois et al., 2003) e com associação de mais de uma ajuda externa (Nolan et al., 2001). Para além disto, verificam-se benefícios da associação de treinos cognitivos e de medicação, cujos resultados demonstram estabilização ou melhoria de défices cognitivos e funcionais (Bottino et al., 2002). No caso do cruzamento de técnicas, o estudo de Bourgeois et al. (2003), conclui que a Recuperação Espaçada (SR - Spaced Retrieval; método de aprender e reter informação pela repetição ao longo de vários períodos de tempo) apresenta mais vantagens, quando comparada com a Hierarquia de Pistas $(\mathrm{CH}-$ Cuering Hierarchy; é uma sequência sistemática e graduada de pistas, segundo determinados protocolos de actuação), na aquisição de novas aprendizagens de indivíduos com demência. Esta conclusão é complementada pelo estudo de Camp et al. (1996), que referem que a SR aliada ao calendário (ajuda externa) promove continuidade do uso desta ajuda externa no follow-up. Para além disto, a associação de mais do que uma ajuda externa, parece ter influência positiva na capacidade dos indivíduos em realizarem com sucesso as tarefas propostas, nomeadamente ao nível da procura e identificação correcta dos elementos do meio (Nolan et al., 2001). Neste sentido, Kapur (2009) refere que um eficiente uso de ajudas externas à memória envolve motivação, paciência, planeamento, capacidade de resolução de problemas, concentração, aprendizagem e a própria memória.

Assim, do ponto de vista da continuidade das melhorias em fases pós-intervenção, Wilson et al. (1997), concluíram que ocorrem grandes variações nos resultados dos participantes. Esta oscilação deverá ser compreendida, na opinião dos autores, em parte pela falta de adequação da introdução de determinada ajuda externa, no quotidiano de um indivíduo, isto é, o envelhecimento não é homogêneo entre os indivíduos, devendo ser avaliado cada sujeito no sentido de se percepcionar qual a melhor estratégia de compensação a introduzir. Neste sentido, o estudo de Clare et al. (2000), vem acentuar a importância de individualizar e ajustar a intervenção, pois o paciente $\mathrm{F}$, que apenas usou ajuda externa electrónica, nunca se habituou complemente à mesma, tendo-a abandonado, logo após a fase de intervenção. É portanto da opinião dos autores que o uso de ajudas externas deve integrar-se na avaliação de necessidades e no planeamento diário das actividades, traduzindo-se numa intervenção com eficácia e com continuidade no follow-up.

A avaliação individual dos sujeitos deve ainda enquadrar-se em situações que o utente se esquece da ajuda externa, da sua localização, não a usa no momento certo ou depende de outros para o recurso a esta (Bourgeois et al., 2003). Deve portanto, realizar-se na promoção da participação do utente no processo de reabilitação, minimizando comportamentos automatizados e fomentando a auto-percepção da eficácia da sua memória.

Segundo Bourgeois (1992), Hanley (1986), Kurlychek (1983), citados por Clare et al., 2000), o treino das ajudas externas à memória em pessoas idosas com demência, tem sido investigado num pequeno número de estudos, no entanto Bourgeois et al. (2003), afirma que desde os anos 90 o número de estudos publicados, documentando a eficácia das intervenções em indivíduos com demência, têm aumentado bastante, tendo sido testados mais ao nível das lesões cerebrais e da demência, e os resultados demonstram eficácia no follow-up, como refere o estudo de Wilson et al. (1997).

\section{Discussão}

As ajudas externas enquadram-se como mecanismos de compensação, que tal como as modificações arquitetônicas e ambientais, permitem a manutenção da memória, orientação espácio-temporal e segurança da pessoa idosa com demência, numa perspectiva de delineação de um espaço familiar, pessoal e individual (Barbosa \& Martín, 2008). Devem ainda ser referenciadas e utilizadas como mecanismos de recordação de acções futuras, salvaguardando-se a memória prospectiva e promotoras da memória retrospectiva, através de mecanismos como câmaras ou diários (Hodges et al., 2006; Kapur, Glisky, \& Wilson, 2004). 
Actualmente o uso de ajudas externas com recurso a tecnologias tem vindo a despoletar interesse na comunidade científica, sendo que no estudo de Wilson et al. (1997) revela-se que esta aliança promoveu o aumento significativo das tarefas realizadas com sucesso pelos indivíduos. Os computadores pessoais têm adquirido grande importância como prótese de memória, nomeadamente em indivíduos com incapacidades mnésicas, no entanto Kapur et al. (2004), referem que os seus benefícios no funcionamento do dia-a-dia, ainda não estão amplamente analisados. Será portanto, na opinião dos autores, importante incluir a avaliação da introdução de ajudas externas com recurso a tecnologia, no plano de cuidados; proporcionar um design mais simplificado e ajustado ao quotidiano, possuir tecnologia eficiente e de fácil utilização e atentar ao custo para o utente na sua aquisição.

Do ponto de vista dos tipos de memória abrangidos pelos vários estudos, poderá dizer-se que, nos 33 estudos analisados, em 11 a memória foi analisada como construto global (memória) e associada às tarefas do dia-a-dia; a memória explicita foi estudada em 4 estudos; implícita em 4 estudos; a prospectiva e retrospectiva ambas apenas em 1 estudo e a operativa em 2. Para além disto, a pesquisa realizada recolheu diferentes tipos de amostras em análise, sendo que em alguns artigos foram analisados grupos de indivíduos com comprometimento mnésico, demência ou MCI em paralelo com grupos de indivíduos sem comprometimento.

Destaca-se a necessidade de repensar os estudos que relacionam o uso de ajudas externas com a estimulação da memória, sendo esta análise necessária aos diferentes subtipos de memória, e tendo em linha de conta, os diferentes grupos envolvidos.

\section{Considerações Finais}

$\mathrm{Na}$ intervenção o primeiro passo deve ser baseado na avaliação de necessidades dos indivíduos, tendo em linha de conta aspectos como a componente cultural, individual, habilitações literárias, condicionantes de saúde e psicossociais. Esta etapa de avaliação e conhecimento, deve ser utilizada em indivíduos sem e com comprometimento cognitivo, como o caso da demência. No caso das pessoas idosas com comprometimento cognitivo ao nível de falhas mnésicas, a introdução de estratégias compensatórias, nomeadamente as ajudas externas, têm de ser acompanhadas de um processo de treino e de ajuste às características e necessidades inicialmente identificadas pelos indivíduos. Esta etapa é fulcral, na manutenção e introdução de ajudas externas no quotidiano dos indivíduos, no sentido de se promover a utilização espontânea do seu uso, à medida que os indivíduos têm maiores incapacidades mnésicas. Estes aspectos são relacionados com a cultura e o insight dos indivíduos, isto é, quanto maior for a percepção dos indivíduos do seu comprometimento cognitivo, maior é a tendência para a utilização de ajudas externas.
Nos indivíduos sem comprometimento cognitivo, verifica-se que aumentam a utilização de ajudas externas ao longo do processo de envelhecimento, sendo este caracterizado por sucessivas fases de adaptação contínua às perdas que se vão instalando, é fulcral no entanto, o acompanhamento dos profissionais no desenvolvimento de estratégias de adaptação às incapacidades e estimulação contínua das capacidades individuais.

Em futuros trabalhos será importante ainda analisar em que medida a diferença de géneros é preditiva de maior frequência da utilização de ajudas externas e se há correlação entre o tipo e ajudas externas utilizadas e o género do indivíduo. Devem ser realizados estudos interventivos que avaliem as condições a seguir para manter os sujeitos com altos níveis de utilização de ajudas externas, e ainda estudos de avaliação de quais as ajudas externas mais eficientes para cada patologia/síndrome e características individuais das pessoas idosas.

Como se verifica pela análise dos resultados, a grande maioria dos estudos realizados apostam em metodologias de exploração e não interventivas, sendo que futuramente dever-se-á investigar com maior profundidade as circunstâncias de utilização das ajudas externas enquanto mecanismo compensatório, na intervenção de indivíduos com e sem comprometimento mnésico. Será necessário, esclarecer em ambas as circunstâncias (sem ou com comprometimento mnésico), quais os factores inter-relacionados que influenciam o uso de ajudas externas.

No estudo de Nolan et al. (2001), são aplicadas duas ajudas externas, que foram avaliadas enquanto ajudas complementares, no entanto, será necessário em trabalhos futuros avaliar qual a ajuda que contribui com maior peso, para o aumento de $50 \%$ da capacidade das pessoas idosas localizarem o seu quarto.

É uma linha de investigação prometedora mas que foi limitada em determinados grupos de trabalho nos anos 90 e 2000, e agora será necessário reactivar o seu estudo, mas numa perspectiva ainda mais minuciosa de análise das variáveis e factores em causa. Necessita de uma nova revolução e empenho para prosseguir aprofundadamente com novas pesquisas.

\section{Referências}

American Psychiatric Association. (2000). Diagnostic and statistical manual of mental disorders - DSM-IV-TR $\left(4^{\text {th }} \mathrm{Rev}\right.$. Ed.). Washington, DC: American Psychiatric Press.

Bäckman, L., \& Dixon, R. (1992). Psychological compensation: A theoretical framework. Psychological Bulletin, 112(2), 259-283.

Barbosa, C., \& Martín, J. (2008). Desenho arquitectónico de equipamentos sociais para idosos com demência. In Actas do $7^{\circ}$ Congresso Nacional de Psicologia da Saúde (pp. 552559). Porto, Portugal: Universidade do Porto.

Bottino, C., Carvalho, I., Alvarez, A. M., Avila, R., Zukauskas, P., Bustamante, S., et al. (2002). Reabilitação cognitiva em pacientes com doença de Alzheimer: Relato de trabalho em equipe multidisciplinar. Arquivos de Neuro-Psiquiatria, 60(1), 70-79. 
Barbosa, C. M. O. \& Martin, J. I. G. (2012). Ajudas Externas à Memória na Intervenção em Pessoas Idosas com Comprometimento Mnésico.

Bourgeois, M. (2006). External aids. In D. K. Attix \& K. A. Welsh-Bohmer (Eds.), Geriatric Neuropsychology: Assessment and intervention (pp. 333-346). New York: The Guilford Press.

Bourgeois, M., Camp, C., Rose, M., White B., Malone, M., Carr, J., et al. (2003). A comparison of training strategies to enhance use of external aids by persons with dementia. Journal of Communication Disorders, 36(5), 361-378.

Burns, A., \& Zaudig, M. (2002). Mild cognitive impairment in older people. Lancet, 360, 1963-1965.

Camp, C., Foss, J., O’Hanlon, A., \& Stevens, A. (1996). Memory interventions for persons with dementia. Applied Cognitive Psychology, 10(3), 193-210.

Camp, C., \& Skrajner, M. (2004). Resident-Assisted Montessori Programming (RAMP): Training persons with dementia to serve as group activity leaders. The Gerontologist, 44(3), 426-431.

Clare, L., Wilson, B., Carter, G., Breen, K., Gosses, A., \& Hodges, J. (2000). Intervening with every day memory problems in dementia of Alzheimer type: An errorless learning approach. Journal of Clinical and Experimental Neuropsychology, 22(1), 132-146.

De Frias, C., Dixon, R., \& Bäckman, L. (2003). Use of memory compensation strategies is related to psychosocial and health indicators. Journal of Gerontology: Psychological Sciences, $58 B(1), 12-22$.

De Vreese, L., Neri, M., Fioravanti, M., Belloi, L., \& Zanetti, O. (2001). Memory rehabilitation in Alzheimer's disease: A review of progress. International Journal of Geriatric Psychiatry, 16(8), 794-809.

Dixon, R., \& De Frias, C. (2007). Mild memory deficits differentially affect 6 -year changes in compensatory strategy use. Psychology and Aging, 22(3), 632-638.

Dixon, R., De Frias, C., \& Bäckman, L. (2001). Characteristics of self-reported memory compensation in older adults. Journal of Clinical and Experimental Neuropsychology, 23(5), 650-661.

Dixon, R., Hopp, G., Cohen, A., De Frias, C., \& Bäckman, L. (2003). Self-reported memory compensation: Similar patterns in Alzheimer's disease and very old adult samples. Journal of Clinical and Experimental Neuropsychology, 25(3), 382390.

Evans, J., Wilson, B., Needham, P., \& Brentnall, S. (2003). Who makes good use of memory aids? Results of a survey of people with acquired brain injury. Journal of International Neuropsychological Society, 9(6), 925-935.

Francés, I., Barandiaran, M., Marcellan, T., \& Moreno, L. (2003). Estimulación psicocognoscitiva en las demências. Anales del sistema sanitario de Navarra, 26(3), 405-422.

Gauthier, S., Reisberg, B., Zaudig, M., Petersen, R. C., Ritchie, K., Broich, K., et al. (2006). International Psychogeriatric Association Expert Conference on mild cognitive impairment. Mild cognitive impairment. Lancet, 367(9518), 1262-1270.

Grandmaison, E., \& Simard, M. (2003). A critical review of memory stimulation programs in Alzheimer's disease. Journal Neuropsychiatry Clinic Neuroscience, 15(2), 130144.

Hodges, S., Williams, L., Berry, E., Izadi, S., Srinivasan, J., Butler, A., et al. (2006). SenseCam: A retrospective memory aid. In P. Dourish \& A. Friday (Eds.), Ubicomp 2006, LNCS 4206 (pp. 177-193). Berlin, Germany: Springer Verlag.

Jorm, A. F., Korten, A. E., \& Henderson, A. S. (1987). The prevalence of dementia: A quantitative integration of the literature. Acta Psychiatrica Scandinavica, 76(5), 465-479.
Kapur, N. (2009). Compensating for memory deûcits with memory aids memory. In B. A. Wilson (Eds.), Rehabilitation: Integrating theory and practice. New York: The Guilford Press.

Kapur, N., Glisky, E., \& Wilson, B. (2004). Technological memory aids for people with memory deficits. Neuropsychological Rehabilitation, 14(1/2), 41-60.

Koltai, D., Welsh-Bohmer, K., \& Schmechel, D. (2001). Influence of anosognosia on treatment outcome among dementia patients. Neuropsychological Rehabilitation, 11(3/ 4), 455-475.

Kurz, A., Pohl, C., Ramsenthaler, M., \& Sorg, C. (2009). Cognitive rehabilitation in patients with mild cognitive impairment. International Journal Geriatric Psychiatry, 24(2), 163-168.

Lyketsos, C. G., Steinberg, M., Tschanz, J. T., Norton, M. C., Steffens, D. C., \& Breitner, J. (2000). Mental and behavioral disturbances in dementia: Findings from the cache county study on memory in aging. American Journal of Psychiatry, 157(5), 708-714.

McDougall, G. (1998). Increasing memory self-efficacy and strategy use in Hispanic elders. Clinical Gerontologist: The Journal of Aging and Mental Health, 19(2), 57-76.

Melton, A., \& Bourgeois, M. (2005). Training compensatory memory strategies via the telephone for persons with TBI. Aphasiology, 19(3/5), 353-364.

Nolan, B., Mathews, R., \& Harrison, M. (2001). Using external memory aids to increase room finding by older adults with dementia. American Journal of Alzheimer's Disease and Other Dementias, 16(4), 251-254.

Reese, C., Cherry, K., \& Norris, L. (1999). Practical memory concerns of older adults. Journal of Clinical Geropsychology, 5(4), 231-244

Soler, M., \& Ruiz, J. (1996). The spontaneous use of memory aids at different educational levels. Applied Cognitive Psychology, 10(1), 41-51.

Tran, Q., Mynatt, E., \& Calcaterra, G. (2007). Using memory aid to build memory independence. Proceedings of HCII: Human-Computer Interaction International, 959-965.

Turkstra, L. (2001). Treating memory problems in adults with neurogenic communication disorders. Seminars in Speech and Language, 22(2), 147-155.

Wilson, B. (1997). Cognitive rehabilitation: How it is and how it might be. Journal of the International Neuropsychological Society, 3(5), 487-496.

Wilson, B., Emslie, H., Quirk, K., \& Evans, J. (2001). Reducing everyday memory and planning problems by means of a paging system: A randomised control crossover study. Journal of Neurology, Neurosurgery and Psychiatry, 70(4), 477-482.

Wilson, B., Evans, J., Emslie, H., \& Malinek, V. (1997). Evaluation of NeuroPage: A new memory aid. Journal of Neurology, Neurosurgery and Psychiatry, 63(1), 113-115.

Wilson, B., \& Watson, P. (1996). A practical framework for understanding compensatory behaviour in people with organic memory impairment. Psychology Press, 4(5), 465-486.

Woods, B. (1994). Management of memory impairment in older people with dementia. International Review of Psychiatry, 6(2/3), 153-161. 